\title{
Genomic and functional characterization of five novel Salmonella-targeting bacteriophages
}

\author{
Marta Kuźmińska-Bajor ${ }^{1 *} \mathbb{0}$, Paulina Śliwka ${ }^{1}$, Maciej Ugorski², Paweł Korzeniowski ${ }^{1}$, Aneta Skaradzińska', \\ Maciej Kuczkowski ${ }^{3}$, Magdalena Narajaczyk ${ }^{4}$, Alina Wieliczko ${ }^{3}$ and Rafał Kolenda ${ }^{2}$
}

\begin{abstract}
Background: The host-unrestricted, non-typhoidal Salmonella enterica serovar Enteritidis (S. Enteritidis) and the serovar Typhimurium (S. Typhimurium) are major causative agents of food-borne gastroenteritis, and the host-restricted Salmonella enterica serovar Gallinarum (S. Gallinarum) is responsible for fowl typhoid. Increasing drug resistance in Salmonella contributes to the reduction of effective therapeutic and/or preventive options. Bacteriophages appear to be promising antibacterial tools, able to combat infectious diseases caused by a wide range of Salmonella strains belonging to both host-unrestricted and host-restricted Salmonella serovars.
\end{abstract}

Methods: In this study, five novel lytic Salmonella phages, named UPWr_S1-5, were isolated and characterized, including host range determination by plaque formation, morphology visualization with transmission electron microscopy, and establishment of physiological parameters. Moreover, phage genomes were sequenced, annotated and analyzed, and their genomes were compared with reference Salmonella phages by use of average nucleotide identity, phylogeny, dot plot, single nucleotide variation and protein function analysis.

Results: It was found that UPWr_S1-5 phages belong to the genus Jerseyvirus within the Siphoviridae family. All UPWr_S phages were found to efficiently infect various Salmonella serovars. Host range determination revealed differences in host infection profiles and exhibited ability to infect Salmonella enterica serovars such as Enteritidis, Gallinarum, Senftenberg, Stanley and Chester. The lytic life cycle of UPWr_S phages was confirmed using the mitomycin C test assay. Genomic analysis revealed that genomes of UPWr_S phages are composed of 51 core and 19 accessory genes, with 33 of all predicted genes having assigned functions. UPWr_S genome organization comparison revealed 3 kinds of genomes and mosaic structure. UPWr_S phages showed very high sequence similarity to each other, with more than 95\% average nucleotide identity.

Conclusions: Five novel UPWr_S1-5 bacteriophages were isolated and characterized. They exhibit host lysis range within 5 different serovars and are efficient in lysis of both host-unrestricted and host-restricted Salmonella serovars. Therefore, because of their ability to infect various Salmonella serovars and lytic life cycle, UPWr_S1-5 phages can be considered as useful tools in biological control of salmonellosis.

Keywords: Salmonella enterica, Bacteriophages, Bacteriophage genetics, Comparative genomics

\footnotetext{
*Correspondence: marta.kuzminska-bajor@upwr.edu.pl

${ }^{1}$ Department of Biotechnology and Food Microbiology, Faculty

of Biotechnology and Food Sciences, Wrocław University

of Environmental and Life Sciences, Wrocław, Poland

Full list of author information is available at the end of the article
}

\section{Background}

Salmonella enterica is one of the major causative agents of human gastrointestinal infections from contaminated food of animal origin. The severity of salmonellosis in original author(s) and the source, provide a link to the Creative Commons licence, and indicate if changes were made. The images or other third party material in this article are included in the article's Creative Commons licence, unless indicated otherwise in a credit line to the material. If material is not included in the article's Creative Commons licence and your intended use is not permitted by statutory regulation or exceeds the permitted use, you will need to obtain permission directly from the copyright holder. To view a copy of this licence, visit http://creativecommons.org/licenses/by/4.0/. The Creative Commons Public Domain Dedication waiver (http://creativeco mmons.org/publicdomain/zero/1.0/) applies to the data made available in this article, unless otherwise stated in a credit line to the data. 
humans varies from mild symptoms to life-threatening conditions, depending on various factors including the properties of the infecting serovars and their host specificity [1-3]. Two host-unrestricted Salmonella enterica serovars, Enteritidis and Typhimurium, commonly isolated from poultry products, are the most frequent causes of acute gastroenteritis $[4,5]$. Therefore, continuous growth of poultry eggs and meat production and consumption lead to increasing public health threats. On the other hand, the host-restricted Salmonella enterica serovar Gallinarum infecting only avian species causes fowl typhoid characterized by high morbidity and mortality, which often leads to a severe septicemic disease, and is responsible for considerable economic losses in the poultry industry worldwide [6,7].

A major worldwide problem in combatting salmonellosis is the rapidly growing antibiotic resistance [8]. The prevalence of antibiotic-resistant Salmonella strains poses a serious threat to public health as a source of untreatable infections and epidemics. Therefore, it is necessary to develop and apply new strategies to prevent and control these infections. One such approach is phage therapy, proposed as an important alternative to antibiotic treatment and as a preventive strategy in human infections and food production $[9,10]$. Bacteriophages are self-replicating and self-limiting bacterial viruses since they multiply only at the site where the host is located and are eliminated gradually in the absence of host bacteria [11]. Low inherent toxicity, lack of cross-resistance with antibiotics and versatility are the advantages of phages in both therapy and food safety $[12,13]$. However, bacteriophages are host-specific and often infect only one $S$. enterica serovar $[14,15]$, which in many instances may be a limiting factor in pathogen elimination, as new phages have to be identified for each serovar or even strain causing an epidemic or outbreak [16]. Therefore, bacteriophages used as a preventive strategy or as an antimicrobial tool should show lytic activity against a wide range of pathogens. In the case of Salmonella, such broad-host-range bacteriophages were described as those that infected at least several serovars. However, the term broad-host-range Salmonella phages was used for phages able to infect 27 Salmonella serovars [17] as well as only three Salmonella serovars [18]. It was also shown that Salmonella phages were able to lyse Escherichia coli and Klebsiella oxytoca strains [19] as well as strains belonging to the species Enterobacter cloacae and Cronobacter sakazakii [20].

Considering the great potential of bacteriophages as antimicrobial agents in Salmonella eradication, this study was undertaken to isolate and characterize bacteriophages against a wide spectrum of $S$. enterica serovars. As a result, five novel bacteriophages, named
UPWr_S1-5, able to infect numerous Salmonella strains, including these belonging to the host-unrestricted serovar $S$. Enteritidis and host-restricted $S$. Gallinarum were isolated and characterized.

\section{Material and methods \\ Bacterial strains, phages and growth conditions}

Salmonella enterica strains (63) used in this study were obtained from the Strain Collection of the Department of Epizootiology and Clinic of Bird and Exotic Animals, Wrocław University of Environmental and Life Sciences. All bacterial strains were cultivated in LuriaBertani (LB) broth (Sigma-Aldrich, Germany) under aerobic conditions at $37{ }^{\circ} \mathrm{C}$ with agitation. The lysogenic phage P22 (DSM 18523), the strictly lytic phage Felix O1 (DSM 18523) and S. Typhimurium LT2 (DSM 18522) as their host were obtained from the German Collection of Microorganisms and Cell Cultures GmbH (DSMZ, Braunschweig, Germany). The phages and $S$. Typhimurium LT2 reconstitution and propagation were conducted in accordance with the supplier's instructions.

\section{Bacteriophage isolation and purification}

One hundred eighty-four samples, including feces, litter and manure from poultry farms, drainage ditches located near poultry farms or treatment plants were collected from 2015 to 2016. For bacteriophage isolation, $5 \mathrm{~g}$ of a solid sample or $5 \mathrm{ml}$ of a liquid sample was mixed with $15 \mathrm{ml}$ of LB broth, inoculated with five randomly chosen Salmonella strains and incubated overnight at $37^{\circ} \mathrm{C}$ with agitation. Bacterial cultures were centrifuged and supernatants containing phages were filtered using a $0.22 \mu \mathrm{m}$ filter (Merck Millipore, USA). The presence of phages was assessed using a spot test. For this purpose, overnight cultures of Salmonella were spread on LB agar plates and incubated for $40 \mathrm{~min}$ at room temperature. Serial dilutions of filtered supernatants containing phages were spotted onto the surface of the plates, left to dry and incubated at $37{ }^{\circ} \mathrm{C}$ overnight or until a visible bacterial lawn grew. Plates were inspected for lysis zones or the presence of plaques. Clear, single and wellseparated plaques were picked and eluted into $200 \mu \mathrm{l}$ of LB broth culture. Phage suspension was added to $5 \mathrm{ml}$ of the fresh host culture and incubated overnight. To obtain a single phage preparation, each bacteriophage was purified using five consecutive rounds of single-plaque picking and propagation.

\section{Bacteriophage amplification and titer determination}

On the basis of clear plaque formation and efficient propagation, $S$. Enteritidis A41 and A28 were used as hosts for phages UPWr_S1 and UIPWr_S5, respectively, whereas S. Enteritidis A36 was employed as a host for UPWr_S2, 
UPWr_S3 and UPWr_S4. The propagation of isolated bacteriophages was performed on the respective host strain. The phages were propagated according to Oliveira et al. [21] with slight modifications. The bacterial culture was prepared by inoculation of $10 \mathrm{ml}$ of LB broth with a single colony following overnight incubation at $37^{\circ} \mathrm{C}$ with agitation. In short, $0.5 \mathrm{ml}$ of overnight culture was inoculated into $10 \mathrm{ml}$ of fresh LB broth and cultivated for $2 \mathrm{~h}$. Then, $5 \mathrm{ml}$ of phage suspension was added and culture was continued overnight at $37^{\circ} \mathrm{C}$. In the next step, the bacterial culture was centrifuged for $10 \mathrm{~min}$ at $5000 \times g$ to remove any remaining cell debris and filtered through $0.22 \mu \mathrm{m}$ pore size syringe filters. The resulting phage suspension was again added to $100 \mathrm{ml}$ of fresh host culture and incubated overnight at $37^{\circ} \mathrm{C}$. The centrifugation and filtration steps were repeated. Bacteriophage titer was determined using routine test dilution and double-agar overlay [22].

\section{Host-range determination and efficiency of plating}

To evaluate the lytic spectrum of the isolated bacteriophages, a spot test was employed by dropping high titer phage lysates $\left(10^{12}-10^{14}\right.$ plaque forming units $\left.(\mathrm{PFU}) / \mathrm{ml}\right)$ onto agar plates with Salmonella strains. The plates were incubated at $37^{\circ} \mathrm{C}$ for $24 \mathrm{~h}$ and examined for the degree of clearing zones.

For efficiency of plating (EOP), the spot test was conducted with phage lysates diluted serially 10 times and spotted on agar plates with phage-sensitive Salmonella strains [23]. The EOP was calculated as the ratio of PFU formed by phages infecting Salmonella strains to PFU formed on a propagation host. All the experiments were carried out in triplicate. EOP values were defined as high when $\mathrm{EOP} \geq 0.5$, moderate when $0.01 \leq \mathrm{EOP}<0.5$ and low when $0.0001<\mathrm{EOP}<0.01$ [24]. The data were reordered by hierarchical clustering analysis using the complete linkage method and R software [25]. Next, a heatmap was generated with the package ggplot2 implemented in the $\mathrm{R}$ software [26]. The $\mathrm{R}$ code is provided in Additional file 1: File S1.

\section{Morphological classification}

To ascertain the morphology of high titer phage samples, $5 \mu \mathrm{l}$ of phage suspension was adsorbed onto 400 copper mesh grids (Sigma-Aldrich) coated with $2 \%$ collodion solution (Sigma-Aldrich) and carbon for $3 \mathrm{~min}$, stained with $2 \%$ uranyl acetate (pH 4.5) (BDH Chemicals, UK) for $15 \mathrm{~s}$ and air-dried. Electron microscopic analysis was performed at $120 \mathrm{kV}$ using a Tecnai G2 Spirit BioTWIN transmission electron microscope (FEI). Micrographs were taken at 250,000 times magnification, with Olympus Soft Imaging Solution software.

\section{Mitomycin C assay}

In order to determine the phage life cycle, the mitomycin $\mathrm{C}$ assay was performed according to Owen et al., [27] with some modifications. Briefly, for prophage induction, the temperate phage P22, the strictly lytic phage Felix $\mathrm{O} 1$ and their host $S$. Typhimurium LT2 were used as positive and negative controls, respectively. Phages were spotted on the fresh bacterial lawns of respective hosts and incubated overnight at $37{ }^{\circ} \mathrm{C}$. After incubation, the presence of resistant bacterial clones was observed in lytic zones. At least 10 phageresistant colonies were picked from one plate and purified by fivefold subculturing on MacConkey agar (Sigma-Aldrich) in order to remove attached phage particles. To confirm phage resistance of these bacterial strains, a standard spot test was performed. For chemical induction of phages from phage-resistant strains, $100 \mathrm{ml}$ of LB broth was inoculated with overnight bacterial cultures and cultivated until an optical density at $600 \mathrm{~nm}$ of 0.2 was reached. To stimulate prophage induction, mitomycin C (Sigma-Aldrich) was added to the final concentration of $1 \mu \mathrm{g} / \mathrm{ml}$. As a negative control each of the analyzed bacterial cultures was grown in the absence of mitomycin C. Overnight cultures, both with and without mitomycin $C$ induction, were centrifuged at $4,000 \times \mathrm{g}$, filtered through $0.22 \mu \mathrm{m}$ filter and spotted on cultured Petri dishes with the appropriate Salmonella host. After overnight incubation at $37^{\circ} \mathrm{C}$, plates were analyzed for the presence of clear zones.

\section{One-step growth curve, latent period and burst size}

The adsorption assay was carried out according to Rahman et al. [28] with minor modifications. The log phase cultures of host strains were infected with phage suspensions at optimal multiplicity of infection (MOI) and incubated at $37{ }^{\circ} \mathrm{C}$. At $0,3,6,9,12,15$, and $18 \mathrm{~min}$ of incubation, aliquots were taken $(100 \mu \mathrm{l})$, mixed with LB medium $(900 \mu \mathrm{l})$, and immediately filtered through a $0.22 \mu \mathrm{m}$ pore size syringe filter. The titer of unadsorbed phage particles was determined by the double-agar layer method. The experiment was repeated three times for each phage.

To determine the latent period and phage burst size, a one-step growth curve was performed according to Yu et al. [29]. Phage suspensions were mixed with exponential growth phase cultures of host bacteria at optimal MOI. Samples were taken at $10 \mathrm{~min}$ intervals over a period of $80 \mathrm{~min}$ and phage titer was determined via the double-agar layer method. Latent time was measured as the interval between phage adsorption and the liberation of phage particles. Burst size was calculated as the ratio of the phage titer at the plateau phase to the initial count 
of infected bacterial cells/initial phage titer. Each experiment was performed in triplicate.

\section{Adsorption curve}

To determine the adsorption rate, Salmonella strains were grown in LB medium to the exponential phase, then infected with UPWr_S1, UPWr_S4 and UPWr_S5 phages at MOI 1 and UPWr_S2 and UPWr_S3 phages at MOI 0.1 and incubated at $37{ }^{\circ} \mathrm{C}$. Samples were taken at 1,2 , $3,4,5,6,7,8,9,10,15$ and $18 \mathrm{~min}$ and centrifuged. The supernatants were used for plaque assays to determine the titers of non-adsorbed phages [30]. This experiment was repeated three times independently.

\section{Bacteriophage DNA extraction, genome sequencing, assembly and annotation}

High-titer phage suspensions $\left(10^{12}-10^{14} \mathrm{PFU} / \mathrm{ml}\right)$ were used for DNA extraction. Prior to the extraction, bacterial lysates containing phages were treated with DNase I (80 U/ml; Thermo Scientific, USA) and RNase I ( $80 \mu \mathrm{g} /$ $\mathrm{ml}$; Thermo Scientific, USA) at $37{ }^{\circ} \mathrm{C}$ for $3 \mathrm{~h}$ to remove non-phage nucleic acids. Phage DNA was then extracted using a High Pure Viral Nucleic Acid Large Volume Kit (Roche, Mannheim, Germany) with initial phage capsid disruption by treatment with proteinase $\mathrm{K}$ and $0.5 \%$ sodium dodecyl sulfate (Sigma-Aldrich) for $2 \mathrm{~h}$ at $56{ }^{\circ} \mathrm{C}$. The integrity of the extracted DNA was determined by electrophoresis in $0.7 \%$ agarose stained with Midori Green DNA Stain (Nippon Genetics Europe, Germany). The concentration of DNA was determined with a Biowave II UV/Vis spectrophotometer (Biochrom WPA, UK) and purity was determined in terms of the ratio 260/280 nm. Phage genomes were sequenced with the Illumina MiSeq next-generation sequencing platform (Genomed SA. Poland) using MiSeq Reagent Kit v2 500-cycles (Illumina, USA). Sequencing quality was assessed on the basis of average base quality, GC content and adapter contamination [31]. All sequenced phages were assembled into one unique contig and sequence assembly was conducted with the Shovill pipeline and assembly improvement pipeline [32]. Genome assemblies were annotated with Prokka [33].

\section{Comparison, clustering and analysis of phage genomes}

Phage genomes were characterized by overall genome BLAST similarities to the 95 Salmonella phage genomes available at the NCBI. The average nucleotide identity (ANI) of the phage genomes was analyzed using PYANI (v0.2.9) [34]. Genome sequence comparisons were generated with BLASTn and visualized with EasyFig software [35]. The predicted functions of the open reading frames (ORFs) were analyzed for UPWr_S1, UPWr_S2 and UPWr_S5 phages by BLASTn [36] and BLASTp [37] searches, with a cut-off $E$ value of $10^{-4}$. Putative protein sequences were analyzed by BLAST/HMMer Pfam [38] description and Phyre 2.0/HHpred prediction [39, 40] for conservative domain identification. The search of putative tRNA-encoding genes was conducted using ARAGORN [41] as part of the Prokka annotation process [42]. For evolutionary relationships, 60 available genomes of bacteriophages belonging to Jerseyvirus and six available genomes of Cornellvirus bacteriophages, both members of the Guernseyvirinae subfamily, Siphoviridae family, and 29 representative genomes from other phage genera infecting Salmonella were selected from the GenBank Virus database. In contrast to phages from Jerseyvirus and Cornellvirus genera within Guernseyvirinae subfamily, phages belonging to genus Kagunavirus due to their specificity to Escherichia coli were not included in the analysis. All phages used in the analysis are listed in Additional file 2: Table S1. All pairwise comparisons of the nucleotide sequences were conducted using the Genome-BLAST Distance Phylogeny (GBDP) method under settings recommended for prokaryotic viruses, using VICTOR software. The resulting intergenomic distances were used to infer a balanced minimum evolution tree with branch support via FASTME including subtree pruning and regrafting (SPR) postprocessing. Branch support was inferred from 100 pseudo-bootstrap replicates each. Information about genus and family for each sequence was added using iTOL $[43,44]$. The whole genomes were also compared using a dot plot analysis implemented in FlexiDot [45].

\section{Sequence accession numbers}

The annotated UPWr_S phage genome sequences were deposited in GenBank under accession numbers MT588083, MT632017, MT632018, MT632019 and MT632020 for UPWr_S1, UPWr_S2, UPWr_S3, UPWr S4 and UPWr_S5, respectively.

\section{Results}

Bacteriophage host range and EOP determination

A total of 161 isolated phages, named collectively UPWr_S, were tested against 64 Salmonella strains representing 10 different $S$. enterica serovars. Five phages with strong lytic activity and infecting the largest number of Salmonella strains compared to the rest of the analyzed phages, named UPWr_S1-5, were chosen for further studies (Fig. 1). Phages UPWr_S2 and UPWr_S3 lysed 51 and 53 S. enterica strains, respectively. Bacteriophage UPWr_S3 was effective against all 30 analyzed $S$. Enteritidis strains, with 24 strains highly sensitive to phage infection $(E O P \geq 0.5)$. 8/11 $S$. Typhimurium strains (73\%) were lysed in the process of lysis from without, as were 10/11 S. Gallinarum strains (91\%), 
with 9 strains being infected with high effectiveness $(E O P \geq 0.5)$. This phage was able to lyse representatives of $S$. Senftenberg $(E O P \geq 0.5), S$. Chester and $S$. Stanley $(0.0001<\mathrm{EOP}<0.01)$. The phage UPWr_S3 did not infect $S$. Kentucky, S. Mbandaka, S. Infantis or $S$. Newport. UPWr_S2 exhibited a similar host range profile, with a decreased ability to lyse $S$. Typhimurium strains (7/11) in the lysis from without process, and an inability to infect $S$. Chester, $S$. Infantis, $S$. Newport, $S$. Kentucky or $S$. Mbandaka. UPWr_S1 was the only UPWr_S phage which in addition to the majority of $S$. Enteritidis strains $(81 \%$ with $\mathrm{EOP} \geq 0.5)$ infected all tested $S$. Gallinarum strains (10 with $E O P \geq 0.5)$ and $S$. Senftenberg with $\mathrm{EOP} \geq 0.5$, and did not exhibit lytic activity against strains belonging to the remaining serovars. The phage UPWr_S5 showed lytic activity similar to UPWr_S1, and infected $64 \%$ of $S$. Enteritidis and $88 \%$ of $S$. Gallinarum strains with $E O P \geq 0.5$. Finally, the phage UPWr_S4 lysed with high effectiveness (EOP $\geq 0.5) 58 \%$ of $S$. Enteritidis strains and $88 \%$ of $S$. Gallinarum strains. Similar to other characterized phages, UPWr_S4 was able to infect the $S$. Senftenberg strain $(\mathrm{EOP} \geq 0.5)$ but did not lyse $S$. Typhimurium strains or strains from the rest of the analyzed serovars.

Taken together, all UPWr_S phages showed a high ability to infect the majority of tested Salmonella strains belonging to Enteritidis and Gallinarum serovars, and all of them infected $S$. Senftenberg. Only the phages UPWr_S2 and UPWr_S3 could lyse the majority of strains belonging to $S$. Typhimurium nonspecifically, utilizing the lysis from without mechanism, and $S$. Stanley. $S$. Chester strains were lysed only by UPWr_S3.

\section{Assessment of life cycle and genome analysis revealed the lytic infection cycle}

Chemical treatment of lysogenic strains with mitomycin $C$ is known to cause induction of prophages (Owen et al., 2017). All UPWr_S phages produce clear plaques on their Salmonella hosts (Additional file 3: Fig. S1). In order to show that UPWr_S1-5 phages undergo the lytic cycle, induction of Salmonella host strains with mitomycin $\mathrm{C}$ was performed. It was found that all analyzed UPWr_S phage genomes did not contain any mitomycin $\mathrm{C}$-inducible prophages and develop the lytic life cycle. As the positive control, $S$. Typhimurium LT2 colonies resistant to lysogenic phage P22 were treated with mitomycin $C$, yielding plaques with a turbid center at a titer of about $10^{7} \mathrm{PFU} / \mathrm{ml}$, indicating effective prophage induction. In contrast, $S$. Typhimurium LT2 colonies resistant to strictly lytic Felix O1 treated with mitomycin C did not form plaques, indicating the lack of inducible prophages. Moreover, genome analysis revealed that UPWr_S1-5 phage genomes did not encode known integrase or excisionase; hence it is likely that these phages proceed through the lytic life cycle.

\section{Phage morphology}

To classify the Salmonella UPWr_S1-5 phages into morphotype-specific groups, transmission electron microscopy (TEM) was employed and Salmonella bacteriophage particles were examined at 250,000 magnification. All five UPWr_S phages exhibited the B1 morphotype with isometric capsids (ca. 50-57 nm), long tails (length: ca. $112-124 \mathrm{~nm}$ ) and clearly visible tail fibers (Fig. 2). Morphological features are described in Table 1. Morphological analysis of phages showed that they can be classified as members of the genus Jerseyvirus within the Siphoviridae family.

\section{One-step growth curve bacteriophages}

One-step growth curve analysis of UPWr_S phages was performed to determine the latent period and relative burst size per infected bacterial cell. Data generated were analyzed and used to construct the one-step growth curve (Additional file 4: Fig. S2). The latent period of UPWr_S1 was assigned to be $15 \mathrm{~min}$, whereas for phages UPWr_S2 and UPWr_S3 calculated latent periods were 12 and $9 \mathrm{~min}$, respectively. UPWr_S4 and UPWr_S5 phages had the longest latent periods, 24 and $25 \mathrm{~min}$, respectively (Table 1 ). The phage UPWr_S1 had the largest burst size per infected bacterium (201 PFU), while the phages UPWr_S2, UPWr_S3 and UPWr_S5 had a smaller burst size (89, 92 and 92 PFU, respectively), and UPWr S4 had the smallest burst size per infected bacterium (60 PFU).

\section{Determination of adsorption rate}

Adsorption studies were performed to identify the adsorption rate of UPWr_S phages on host bacteria. According to the phage adsorption assay, 90, 93, 99, 80

(See figure on next page.)

Fig. 1 Bacteriophage host range and EOP. Bacteriophages were tested for host ranges and EOP against 64 Salmonella strains belonging to 10 serovars. Obtained results were rearranged using hierarchical clustering analysis and plotted as a heatmap. Bacteriophages used in this analysis are presented on the $\mathrm{X}$ axis and the Salmonella strains are listed on the $\mathrm{Y}$ axis. Each rectangle shows the lytic effect of one bacteriophage to one host and the colors correspond to the lysis pattern: purple, EOP $\geq 0.5$; red, $0.01 \leq \mathrm{EOP}<0.5$; orange, $0.0001<\mathrm{EOP}<0.01$; yellow, lysis from without; white, no lysis 
S. Senftenberg Si65

S. Gallinarum A/177/05

S. Gallinarum A/084/04

S. Gallinarum 5/01

S. Gallinarum 25/06

S. Gallinarum 649/01

S. Gallinarum 629/05

S. Gallinarum 626/05

S. Gallinarum 433/01

S. Gallinarum 383/04

S. Gallinarum 107/01

S. Gallinarum 67/01

S. Enteritidis 327 lux

S. Enteritidis SE71

$S$. Enteritidis SE68

S. Enteritidis SE63

S. Enteritidis SE58

S. Enteritidis SE56

S. Enteritidis SE52

S. Enteritidis SE48

S. Enteritidis SE29

S. Enteritidis S60

S. Enteritidis S59

S. Enteritidis S58

S. Enteritidis S57

S. Enteritidis S56

S. Enteritidis S55

S. Enteritidis S54

S. Enteritidis S53

$\mathrm{S}$. Enteritidis S50

S. Enteritidis S49

S. Enteritidis A42

$\mathrm{S}$. Enteritidis A41

S. Enteritidis A40

S. Enteritidis A39

S. Enteritidis A38

S. Enteritidis A36

S. Enteritidis A33

S. Enteritidis A31

S. Enteritidis A28

$\mathrm{S}$. Enteritidis A23

$\mathrm{S}$. Enteritidis A13

$\mathrm{S}$. Enteritidis A06

$\mathrm{S}$. Enteritidis A05

S. Newport Si43

S. Kentucky Si33

S. Kentucky Si30

S. Infantis Si49

S. Mbandaka Si45

S. Mbandaka Si32

S. Stanley Si31

S. Chester Si47

S. Chester Si53

S. Typhimurium St203

S. Typhimurium St192

S. Typhimurium St185

S. Typhimurium St155

S. Typhimurium St143

S. Typhimurium St105

S. Typhimurium St76

S. Typhimurium St63

S. Typhimurium St42

S. Typhimurium St2

S. Typhimurium LT2

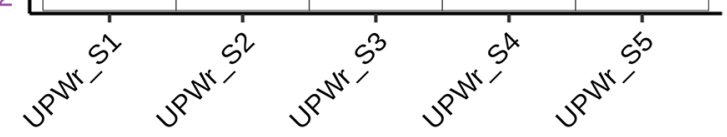

Bacteriophage
EOP

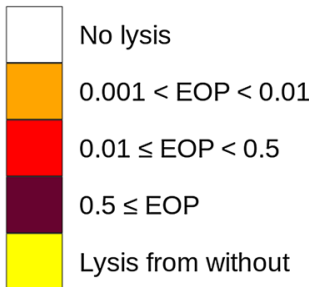

Group

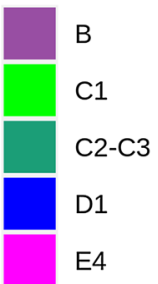

Fig. 1 (See legend on previous page.) 

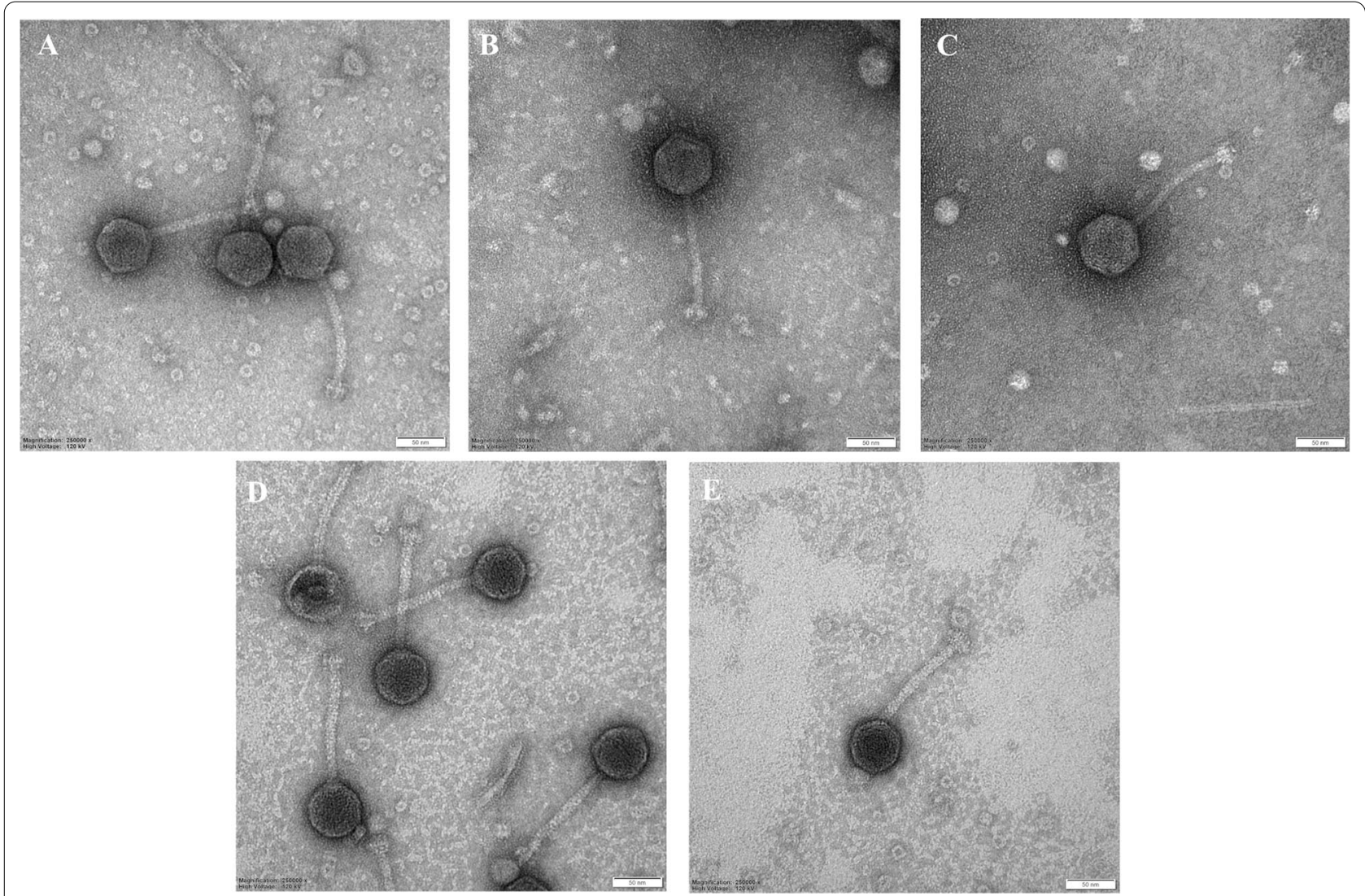

Fig. 2 Transmission electron microscopic (TEM) analysis of UPWr_S phages. All five UPWr_S phages were identified by TEM, UPWr_S1 (a), UPWr_S2 (b), UPWr_S3 (c), UPWr_S4 (d) and UPWr_S5 (e). The black bar represents $50 \mathrm{~nm}$

Table 1 UPWr_S phages' morphological features, genome size and biological characteristics

\begin{tabular}{|c|c|c|c|c|c|}
\hline & UPWr_S1 & UPWr_S2 & UPWr_S3 & UPWr_S4 & UPWr_S5 \\
\hline Capsid size, $\mathrm{nm} \pm \mathrm{SE}^{*}$ & $52 \pm 6$ & $57 \pm 4$ & $52 \pm 5$ & $56 \pm 4.5$ & $51 \pm 4$ \\
\hline Tail size, $\mathrm{nm} \pm \mathrm{SE}$ & $134 \pm 2$ & $136 \pm 7.1$ & $138 \pm 5$ & $129 \pm 4$ & $131 \pm 3.6$ \\
\hline Genome size, bp & 44417 & 44225 & 44154 & 44330 & 44548 \\
\hline GC content, \% & 49.85 & 50.02 & 50.01 & 50.02 & 49.99 \\
\hline $\mathrm{MOI}$ & 1 & 0.1 & 0.1 & 1 & 1 \\
\hline Latent period, min & 15 & 12 & 9 & 24 & 26 \\
\hline Burst size, PFU/cell & 201 & 89 & 92 & 48 & 23 \\
\hline Adsorption degree & 90 & 93 & 99 & 80 & 92 \\
\hline
\end{tabular}

UPWr_S phages' morphological and biological characterization revealed their high degree of similarity. Calculated latent period and burst size for each phage showed the shorter latent period with a higher burst size and lower MOI of phages UPWr_S2 and UPWr_S3

* Head diameter is calculated for isometric capsids. All measurements were made with the program ImageJ. 35 particles were measured for each phage and standard deviation was calculated $( \pm S D)$

** Phage particles adsorbed within $10 \mathrm{~min}$

and 92\% of UPWr_S1, UPWr_S2, UPWr_S3, UPWr_S4 and UPWr_S5, respectively, could adsorb to host bacteria within $10 \mathrm{~min}$ (Table 1), indicating that the phages were readily adsorbed to the host (Additional file 5: Fig. S3).

\section{Characterization of UPWr_S phage genomes}

UPWr_S1-5 phage genomes were sequenced and analyzed on the one hand for their relatedness to each other and, on the other hand, for the presence of any differences. Genome sequencing generated 271,976-408,810 


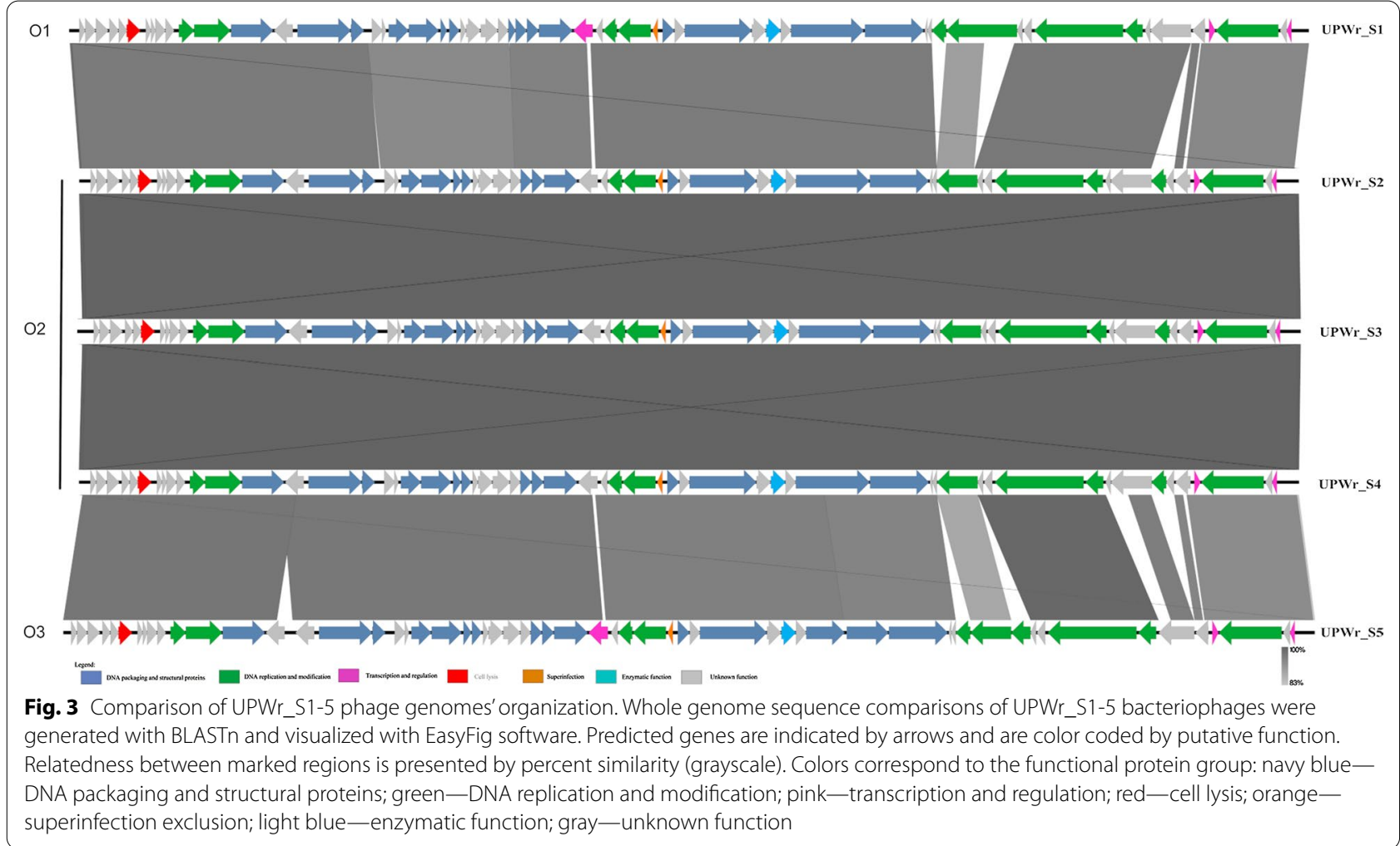

reads for five novel phages with around $1000 \times$ coverage. Analyzed genomes ranged in size from approximately 42 to $43 \mathrm{~kb}$ with $\mathrm{G}+\mathrm{C}$ contents of about $50 \%$. Fifty-one genes, called core genes, were found in all UPWr_S phage genomes, while 19 were not present in all genomes and are called accessory genes. Among them, 14 were of unknown function (Additional file 6: Table S2).

Functional analysis revealed that 14 core genes, present in all 5 genomes, were involved in morphogenesis, coding for capsid, neck, tail fiber, tailspike, capsid decoration and putative tail tape measure proteins. Among accessory genes with predicted morphogenetic function, one gene present in the UPWr_S1 phage genome coded for a fragment of putative head-tail joining protein (gp27) and another gene found in the UPWr_S5 phage genome encoded a fragment of a tail fiber protein (gp70). All of the UPWr_S phage genomes possessed eight core genes responsible for phage replication such as DNA polymerase, recombination endonuclease, putative homing endonuclease, inteins and large and small terminase subunits. Further analysis revealed the presence of three accessory genes involved in phage replication. The genomes of UPWr_S2, UPWr_S3 and UPWr_S4 phages shared one accessory gene encoding a putative protein with homology to homing endonuclease (gp63). In UPWr_S1 and UPWr_S5 phage genomes, DNA primase (gp46) was found. In the genome of the UPWr_S5 phage a gene coding for DNA-cytosine methylase (gp67) taking part in protection from host-encoded exonucleases during phage DNA ejection was found. All UPWr_S phage genomes contained a mobile element such as the putative intein-containing capsid morphogenesis protein (gp15). Another mobile element such as homing endonuclease (gp63) was revealed in UPWr_S2, UPWr_S3 and UPWr S4 phage genomes.

Three regulatory core genes, the gene coding for helix-turn-helix transcriptional regulator (gp58), the gene coding for XRE family transcriptional regulator (gp55), and the gene coding for putative DNA-binding protein (gp31), were detected. Finally, genes facilitating host lysis such as putative lysozyme and holin class II (gp06) and one superinfection immunity protein (gp35) were displayed within core genes and present in all UPWr_S phage genomes. In all UPWr_S genomes, we found the gene encoding enolase (gp40) with unknown function in phages. Our analysis indicated that UPWr_S phages were deprived of known genes encoding toxins, antibiotic resistance or virulence. Also, UPWr_S phage genomes did not contain tRNA genes (Additional file 6: Table S2). Notably, 23 genes representing a significant proportion of core genes were of unknown function. 
Sequence comparison allowed for clear discrimination of 3 kinds of genomes named $\mathrm{O} 1, \mathrm{O} 2$ and $\mathrm{O} 3$ (Fig. 3). Genomes of phages UPWr_S1 and UPWr_S5 represented $\mathrm{O} 1$ and O3, respectively, while UPWr_S2, UPWr_S3 and UPWr_S4 phage genomes all represented the $\mathrm{O} 2$ type.

\section{Phylogenetic analysis revealed close relatedness of UPWr_S phages to the genus Jerseyvirus}

To study the evolutionary relationship of UPWr_S phages, their genomes were compared with previously sequenced Salmonella phages deposited in GenBank. Sixty phages belonging to the Jerseyvirus genus and 35 representatives of other Salmonella phage genera were selected and phylogenetic relationship assessment using VICTOR software was performed. Phylogenetic tree analysis identified three distinct clusters of genomes (Fig. 4). Phage genomes with a high level of genetic similarity belonged to clusters 1 and 2 ( $>50 \%$ bootstrap support). With the exception of the phage St161 (MF158036), all analyzed Jerseyvirus phages belonged to cluster 1 . Cluster 2 was formed by phages belonging to the genus Cornellvirus and phage St161, classified previously as Jerseyvirus; cluster 3 comprised phages representing genera belonging to different families and subfamilies. In contrast to clusters 1 and 2, genomes of phages belonging to cluster 3 were characterized by low relatedness.

The analysis of UPWr_S phage genomes revealed that they belonged to cluster 1 and exhibited close relatedness to phages classified as Jerseyvirus. ANI of phages belonging to cluster 1 was calculated to be $91 \%$. On the basis of 95\% DNA sequence identity [46], UPWr_S phages were categorized and assigned to the genus Jerseyvirus (according to ICTV Taxonomy Release \#35: 2019) [47], which includes such well-characterized phages as SS3e (AY730274), SE2 (JQ007353), wksl3 (JX202565), vB_SenS_Ent2 (NC_023608), vB_SenS Ent1 (NC_019539) and vB_SenS_Ent3 (NC_024204), among others [48-52]. UPWr_S phages showed relatedness with Cornellvirus phages from cluster 2 assigned to be approximately $73 \%$ and were completely unrelated to those from cluster 3, which was confirmed in whole-genome dot plot comparison (Additional file 7:
Fig. S4). In this analysis, it was shown that UPWr_S genomes displayed no sequence similarities to phages not belonging to Guernseyvirinae subfamilies. These results are consistent with previous findings that Jersey phages show low sequence similarity to phages that are not in the Siphoviridae family [53].

Whole genome alignment revealed the presence of 4 differences in nucleotide sequences between UPWr_S2 and UPWr_S3, 8 differences in nucleotide sequences between UPWr_S2 and UPWr_S4, and 5 differences in nucleotide sequences between UPWr_S3 and UPWr S4. These differences included substitutions and deletions (Table 2). Substitutions resulted in amino acid alterations or single nucleotide changes in intergenic regions.

\section{Discussion}

There is a great interest in various practical applications of bacteriophages against Salmonella, with the most attention (and most regulatory approvals) focused on their use to improve food safety [54]. They have been proposed as alternatives to antibiotics in animal health, as biopreservatives in food and as tools for detecting pathogenic bacteria throughout the food chain $[55,56]$. Due to the rising numbers of antibiotic-resistant Salmonella strains, bacteriophage therapy appears to be one of the most promising tools combating these pathogens [57]. The success of such treatment is largely dependent on the biological properties of the phages being used. As Salmonella consists of more than 2,500 serovars, infection efficacy and a wide host spectrum are indispensable requirements for Salmonella-targeting phages, and therefore such phages are naturally the most attractive candidates for the treatment of bacterial infections [58]. Temperate bacteriophages exhibit the potential for gene transduction and may be involved in increasing bacterial virulence [59]. Therefore, phages used for environmental, industrial, or medical purposes should undergo only the lytic life cycle to exclude the possibility of horizontal virulence gene transfer [60]. To meet these requirements, five Salmonella bacteriophages, named UPWr_S1-5, were selected and characterized according to their host range and life cycle.

\footnotetext{
(See figure on next page.)

Fig. 4 Phylogenetic tree of Salmonella bacteriophages. A set of genomes representing available bacteriophages belonging to the genus Jerseyvirus and one representative genome from other genera infecting Salmonella were selected from the GenBank Virus database. All pairwise comparisons of the nucleotide sequences were conducted using the Genome-BLAST Distance Phylogeny (GBDP) method under settings recommended for prokaryotic viruses using VICTOR software. The resulting intergenomic distances were used to infer a balanced minimum evolution tree with branch support via FASTME including SPR postprocessing. Branch support was inferred from 100 pseudo-bootstrap replicates each. Branches with bootstrap values below 50 were collapsed and the bootstrap values equal to or above 50 are shown on the remainder of the tree branches. Information about genus and family for each sequence was added using iTOL
} 


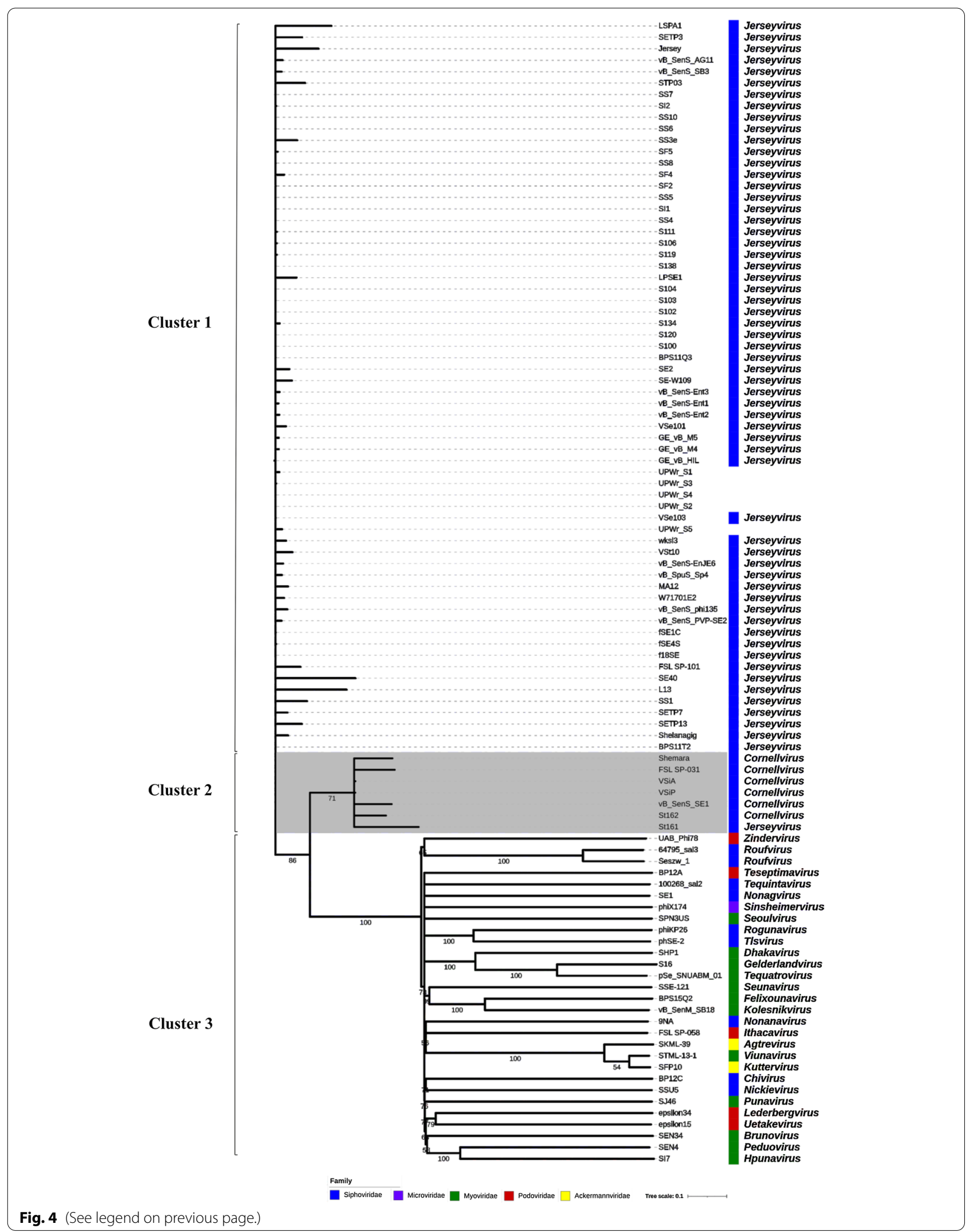


Table 2 Sequence variation between phages UPWr_S2, UPWr_S3 and UPWr_S4

\begin{tabular}{lllll}
\hline Gene product & Protein function & UPWr_S2 vs UPWr_S3 & UPWr_S2 vs UPWr_S4 & UPWr_S3 vs UPWr_S4 \\
\hline gp16 & Putative head decoration protein & - & R132G & R132G \\
gp20 & Putative major capsid protein & - & V338A & V338A \\
Region between gp20-21 & & - & Substitution of g to a & - \\
gp25 & Putative protein & R185W & R185W & - \\
gp30 & Putative tail protein & A94V & - & V94A \\
Region between gp30-gp31 & & Deletion of 1 $t^{* *}$ in UPWr_S2 & Deletion of 1 $t$ in UPWr_S2 & - \\
gp42 & Tail fiber protein & Deletion of 6 AA in UPWr_S2 & Deletion of 6 AA in UPWr_ & - \\
& & $374-379$ (MYKDNG) & S2 374-379 (MYKDNG) & \\
gp43 & Tailspike & - & S2P, G464D & S2P, G464D
\end{tabular}

Whole genome alignment revealed the presence of a few differences in nucleotide sequences between UPWr_S2, UPWr_S3 and UPWr_S4 phages. These differences included deletions and substitutions resulting in amino acid alterations or single nucleotide changes in intergenic regions. There are 4 common substitutions between these phages located in predicted head decoration (gp16), putative major capsid protein (gp20) and tailspike protein with endorhamnosidase function (gp43)

* Letters corresponding to amino acids are written with capital letters

** Letters corresponding to nucleotides are lower case and italicized

- no changes

It was found that UPWr_S1-5 phages belong to the genus Jerseyvirus within the Siphoviridae family and share similar $\mathrm{G}+\mathrm{C}$ and gene content, genome organization and morphology with previously described phages form this genus [48-53]. Members of the genus Jerseyvirus are tailed bacteriophages and infect a number of Salmonella serovars [50-52], with widespread distribution around the world [50] and common isolation from the environment, the most prominent sources being wastewaters [53]. These phages have recently been approved as a safe antiSalmonella zootechnical additive in water for drinking and liquid complementary feed for all avian species known as BAFASAL [61]. In line with these findings, it was observed that UPWr_S1-5 phages infected 3 to 5 Salmonella serovars, including the majority of analyzed $S$. Enteritidis and $S$. Gallinarum clinical strains as well as the frequently isolated $S$. Senftenberg, $S$. Stanley and $S$. Chester strains, which makes them similar to other lytic phages considered to be potential anti-Salmonella agents [17, 62]. Previously, lytic bacteriophages with such a host range were deemed to be suitable anti-Salmonella control agents $[18,51,63]$.

All known members of the proposed genus Jerseyvirus are strictly lytic $[48,51,64]$. Therefore, the lytic life cycle of UPWr_S phages was confirmed using the mitomycin $C$ test assay. It should be mentioned that although some viable Salmonella phages are not inducible using standard techniques including mitomycin $\mathrm{C}$ treatment [65], this induction assay remains an important component of studies aimed at characterization of newly isolated phages. The mitomycin $C$ experimental data were supported by the absence in UPWr_S1-5 phage genomes (similar to other Jersey phages) of int and xis genes coding for integrase and excisionase, respectively, which often play a role in the establishment of lysogeny. Interestingly, UPWr_S1-5 phage genomes contain the gene imm encoding superinfection protein involved in the prevention of infection of already-infected bacteria by other phages [66]. All Jerseyvirus phages contain this gene in their genomes and remain lytic, but the mechanism of this phenomenon remains unknown $[51,67]$.

Another feature of UPWr_S1-5 phages shared with other phages belonging to the genus Jerseyvirus and family Siphoviridae is the mosaic structure of their genomes and presence of mobile elements [49]. Analysis of UPWr phage genes showed the presence of genes coding for an intein and homing endonucleases, which are functionally associated with this phenomenon. Inteins are known to promote the exchange of flanking genes between related Salmonella phages [68], whereas homing endonucleases are DNA-cleaving enzymes that assemble their own reading frames [69], and their activity may lead to mosaicism [70]. It was found that the phages UPWr_S2, UPWr_S3 and UPWr_S4, despite very high genome sequence identity $(>99.9 \%)$ and organization, have slightly different functional characteristics. UPWr_S2 and UPWr_S3 exhibited a shorter latent period and larger burst size than UPWr_S4 and other Jersey phages such as Ent1 [50] and wksl3 [51]. As these parameters play an important role in the host lysis system [71], it suggests that UPWr_S2 and UPWr_ S3 can be considered as useful anti-Salmonella agents.

\section{Conclusions}

In this study, we isolated and characterized five novel UPWr_S1-5 bacteriophages, which were classified in the genus Jerseyvirus within the Siphoviridae family. 
UPWr_S1-5 phages infected gastroenteritis-causing $S$. Enteritidis and the etiological factor of fowl typhoid, $S$. Gallinarum. Therefore, because of their ability to infect various Salmonella serovars and lytic life cycle, they can be considered as useful tools in biological control of salmonellosis.

\section{Patents}

The phages are part of a Wrocław University of Environmental and Life Sciences patent pending. Poland Patent Application P.430168.

\author{
Abbreviations \\ ANI: Average nucleotide identity; GBDP: Genome-BLAST Distance Phylog- \\ eny; DSMZ: German Collection of Microorganisms and Cell Cultures GmbH; \\ NCBI: National Center for Biotechnology Information; LB: Luria-Bertani; ORF: \\ Open reading frame; PFU: Plaque forming unit; TEM: Transmission electron \\ microscopy.
}

\section{Supplementary Information}

The online version contains supplementary material available at https://doi. org/10.1186/s12985-021-01655-4.

Additional file 1: File S1. R code to analyse and plot UPWr_S phages host ranges and EOP and construct a heatmap. For host range determination 64 Salmonella strains belonging to 10 serovars were tested. UPWr_S phages showed a high ability to infect the majority of tested Salmonella strains belonging to Enteritidis and Gallinarum serovars, and all of them infected S. Senftenberg. Only the phages UPWr_S2 and UPWr_S3 could lyse the majority of strains belonging to S. Typhimurium nonspecifically, utilizing the lysis from without mechanism, and S. Stanley. S. Chester strains were lysed only by UPWr_S3.

Additional file 2. Table S1. List of phages used in a comparison study to construct the phylogenetic tree in Fig. 4 and their order in the phylogenetic tree. For evolutionary relationships, 60 available genomes of bacteriophages belonging to Jerseyvirus and six available genomes of Cornellvirus bacteriophages, both members of the Guernseyvirinae subfamily, Siphoviridae family, and 29 representative genomes from other phage genera infecting Salmonella, were selected from the GenBank Virus database.

Additional file 3. Fig. S1: UPWr_S1 (a), UPWr_S2 (b), UPWr_S3 (c), UPWr_S4 (d) and UPWr_S5 (e) phages' plaque morphology on Salmonella Enteritidis lawn. Analysis of plaque morphology revealed that the plaque morphology of each phage was similar, with medium size and a light halo around them. Diameters of plaques were measured manually and diameters of plaques for phages UPWr_S1, UPWr_S2, UPWr_S3, UPWr_S4 and UPWr_S5 were $1.04 \mathrm{~mm}+/-0.28 \mathrm{~mm}, 1.71 \mathrm{~mm}+/-0.21 \mathrm{~mm}, 1.72$ $\mathrm{mm}+/-0.26 \mathrm{~mm}, 1.58 \mathrm{~mm}+/-0.32 \mathrm{~mm}, 2.81 \mathrm{~mm}+/-0.32 \mathrm{~mm}$, respectively.

Additional file 4. Fig. S2: One-step growth curves of UPWr_S phages. The one-step growth curve of UPWr_S phages propagated on their respective hosts in LB medium revealed that the latent periods and burst sizes were approximately 15, 12, 9, 24, 23 and 92 minutes for phages (a) UPWr_S1, (b) UPWr_S2, (c) UPWr_S3, (d) UPWr_S4 and (e) UPWr_S5, respectively. The average burst size was estimated to be 201, 89, 92, 48, 92 PFU/ cell for phages UPWr_S1, UPWr_S2, UPWr_S3, UPWr_S4 and UPWr_S5, respectively.

Additional file 5. Fig. S3: Adsorption curves of UPWr_S phages. Adsorption assays showed that the adsorption rates within 10 minutes for phages (a) UPWr_S1, (b) UPWr_S2, (c) UPWr_S3, (d) UPWr_S4 and (e) UPWr_S5 were $90,93,99,80$ and $92 \%$, respectively.
Additional file 6. Table S2: Predicted ORFs and genes encoded by the UPWr_S phage genomes. Protein sequences of the predicted ORFs of UPWr_S phages were subjected to the BLASTp program to analyze their best known matches on the NCBI website (https://blast.ncbi.nlm.nih.gov). The average nucleotide identity and query coverage were calculated by BLASTp with the cutoff E-value set at 1E-04. "Related phages" refers to a top hit from NCBI BLASTp.

Additional file 7. Fig. S4: Polydot plot comparison of newly sequenced genomes with selected Salmonella phages. All-against-all genome sequence dot plot comparisons of UPWr_S1-5 bacteriophages with selected Salmonella phages belonging to clusters 2 and 3 were performed using FlexiDot. When the DNA residues of both sequences match at the same location on the plot, a dot is drawn at the corresponding position. Once the dots have been plotted, they will combine to form lines that correspond to similar fragments of the genomes. On the main diagonal the sequence's alignment with itself is presented.

\section{Acknowledgements}

The authors would like to acknowledge the technical assistance of Justyna Dubiel for her contributions to the isolation and characterization of UPWr_S phages.

\section{Authors' contributions}

MKB isolated the phages, conceived and designed the analysis, obtained funding, and was a major contributor to writing the manuscript; PŚ performed the phage characterization; MU contributed to writing the manuscript; PK performed host range determination; MK and AW provided Salmonella strains; AS verified the analytical methods; MN performed transmission electron microscopy; RK performed bioinformatic analysis and contributed to manuscript writing. All authors read and approved the final manuscript.

\section{Funding}

This study was supported by the National Centre for Research and Development, LIDER program no. LIDER/378/L-6/14/NCBR/2015. The publication is cofinanced under the Leading Research Groups support project from the subsidy increased for the period 2020-2025 in the amount of 2\% of the subsidy referred to in Art. 387 (3) of the Law of 20 July 2018 on Higher Education and Science, obtained in 2019.

\section{Availability of data and materials}

The annotated UPWr_S phage genome sequences were deposited in the NCBI database at [72] under accession numbers MT588083, MT632017, MT632018, MT632019 and MT632020 for UPWr_S1, UPWr_S2,UPWr_S3, UPWr_S4 and UPWr_S5, respectively.

\section{Declarations}

Ethics approval and consent to participate

Not applicable.

\section{Consent for publication}

Not applicable.

\section{Competing interests}

The authors declare that they have no competing interests.

\section{Author details}

${ }^{1}$ Department of Biotechnology and Food Microbiology, Faculty of Biotechnology and Food Sciences, Wrocław University of Environmental and Life Sciences, Wrocław, Poland. ${ }^{2}$ Department of Biochemistry and Molecular Biology, Faculty of Veterinary Medicine, Wrocław University of Environmental and Life Sciences, Wrocław, Poland. ${ }^{3}$ Department of Epizootiology and Clinic of Birds and Exotic Animals, Faculty of Veterinary Medicine, Wrocław University of Environmental and Life Sciences, Wrocław, Poland. ${ }^{4}$ Department of Electron Microscopy, Faculty of Biology, University of Gdansk, Gdansk, Poland.

Received: 6 April 2021 Accepted: 29 August 2021

Published online: 08 September 2021 


\section{References}

1. Kuźmińska-Bajor M, Kuczkowski M, Grzymajło K, Wojciech Ł, Sabat M, Kisiela D, et al. Decreased colonization of chicks by Salmonella enterica serovar Gallinarum expressing mannose-sensitive FimH adhesin from Salmonella enterica serovar Enteritidis. Vet Microbiol. 2012;158.

2. Gayet R, Bioley G, Rochereau N, Paul S, Corthésy B. Vaccination against salmonella infection: the mucosal way. Microbiol Mol Biol Rev. 2017:81.

3. Grzymajlo K, Ugorski M, Suchanski J, Kedzierska AE, Kolenda R, Jarzab A, et al. The Novel Type 1 Fimbriae FimH Receptor Calreticulin Plays a Role in Salmonella Host Specificity. Front Cell Infect Microbiol. 2017;7.

4. Antunes P, Mourão J, Campos J, Peixe L. Salmonellosis: the role of poultry meat. Clin Microbiol Infect. 2016;22

5. Shivaning Karabasanavar N, Benakabhat Madhavaprasad C, Agalagandi Gopalakrishna S, Hiremath J, Shivanagowda Patil G, B Barbuddhe S. Prevalence of Salmonella serotypes S. Enteritidis and S. Typhimurium in poultry and poultry products. J Food Saf. 2020;40:e12852.

6. Shivaprasad HL. Fowl typhoid and pullorum disease. Rev Sci Tech I'OIE. 2000;19.

7. Xiong W, Wang Y, Sun Y, Ma L, Zeng Q, Jiang X, et al. Antibiotic-mediated changes in the fecal microbiome of broiler chickens define the incidence of antibiotic resistance genes. Microbiome. 2018;6.

8. Cohen E, Davidovich M, Rokney A, Valinsky L, Rahav G, Gal-Mor O. Emergence of new variants of antibiotic resistance genomic islands among multidrug-resistant Salmonella enterica in poultry. Environ Microbiol. 2020:22.

9. Romero-Calle D, Guimarães Benevides R, Góes-Neto A, Billington C. Bacteriophages as Alternatives to Antibiotics in Clinical Care. Antibiotics. 2019;8

10. Lewis R, Hill C. Overcoming barriers to phage application in food and feed. Curr Opin Biotechnol. 2020;61.

11. Huh H, Wong S, St. Jean J, Slavcev R. Bacteriophage interactions with mammalian tissue: Therapeutic applications. Adv Drug Deliv Rev. 2019;145.

12. Malik DJ, Sokolov IJ, Vinner GK, Mancuso F, Cinquerrui S, Vladisavljevic GT, et al. Formulation, stabilisation and encapsulation of bacteriophage for phage therapy. Adv Colloid Interface Sci. 2017;249.

13. Principi N, Silvestri E, Esposito S. Advantages and Limitations of Bacteriophages for the Treatment of Bacterial Infections. Front Pharmacol. 2019;10.

14. Ackermann HW, Petrow S, Kasatiya SS. Unusual bacteriophages in Salmonella newport. J Virol. 1974;13.

15. Sevilla-Navarro S, Catalá-Gregori P, Marin C. Salmonella Bacteriophage Diversity According to Most Prevalent Salmonella Serovars in Layer and Broiler Poultry Farms from Eastern Spain. Animals. 2020;10.

16. Hyman P, Abedon ST. Bacteriophage Host Range and Bacterial Resistance. 2010.

17. Li M, Lin H, Jing Y, Wang J. Broad-host-range Salmonella bacteriophage STP4-a and its potential application evaluation in poultry industry. Poult Sci. 2020;99.

18. Thanki AM, Brown N, Millard AD, Clokie MRJ. Genomic characterization of jumbo Salmonella phages that effectively target United Kingdom pigassociated Salmonella serotypes. Front Microbiol. 2019.

19. Bielke L, Higgins S, Donoghue A, Donoghue D, Hargis BM. Salmonella Host Range of Bacteriophages That Infect Multiple Genera. Poult Sci. 2007;86.

20. Gambino M, Nørgaard Sørensen A, Ahern S, Smyrlis G, Gencay YE, Hendrix $\mathrm{H}$, et al. Phage S144, a New Polyvalent Phage Infecting Salmonella spp. and Cronobacter sakazakii. Int J Mol Sci. 2020;21.

21. Oliveira A, Sillankorva S, Quinta R, Henriques A, Sereno R, Azeredo J. Isolation and characterization of bacteriophages for avian pathogenic E. coli strains. J Appl Microbiol. 2009;106.

22. Adams MH. Bacteriophages. Citeseer; 1959.

23. Kutter E. Phage Host Range and Efficiency of Plating. 2009.

24. Petsong K, Benjakul S, Chaturongakul S, Switt A, Vongkamjan K. Lysis Profiles of Salmonella Phages on Salmonella Isolates from Various Sources and Efficiency of a Phage Cocktail against S. Enteritidis and S. Typhimurium. Microorganisms. 2019;7.

25. Großwendt A, Röglin H. Improved Analysis of Complete-Linkage Clustering. 2015.

26. Wickham H. ggplot2. New York, NY: Springer New York; 2009.
27. Owen SV, Wenner N Canals R, Makumi A, Hammarlöf DL, Gordon MA, et al. Characterization of the prophage repertoire of African Salmonella Typhimurium ST313 reveals high levels of spontaneous induction of novel phage BTP1. Front Microbiol. 2017;8 FEB.

28. Rahman M, Kim S, Kim SM, Seol SY, Kim J. Characterization of induced Staphylococcus aureus bacteriophage SAP-26 and its anti-biofilm activity with rifampicin. Biofouling. 2011:27.

29. Yu YP, Gong T, Jost G, Liu WH, Ye DZ, Luo ZH. Isolation and characterization of five lytic bacteriophages infecting a Vibrio strain closely related to Vibrio owensii. FEMS Microbiol Lett. 2013;348:112-9.

30. Hadas H, Einav M, Fishov I, Zaritsky A. Bacteriophage T4 Development Depends on the Physiology of its Host Escherichia Coli. Microbiology. $1997 ; 143$.

31. Wingett SW, Andrews S. FastQ Screen: A tool for multi-genome mapping and quality control. F1000Research. 2018;7.

32. Page AJ, De Silva N, Hunt M, Quail MA, Parkhill J, Harris SR, et al. Robust high-throughput prokaryote de novo assembly and improvement pipeline for Illumina data. Microb Genomics. 2016:2.

33. Seemann T. Prokka: rapid prokaryotic genome annotation. Bioinformatics 2014:30.

34. Pritchard L, Glover RH, Humphris S, Elphinstone JG, Toth IK. Genomics and taxonomy in diagnostics for food security: soft-rotting enterobacterial plant pathogens. Anal Methods. 2016;8.

35. Sullivan MJ, Petty NK, Beatson SA. Easyfig: a genome comparison visualizer. Bioinformatics. 2011:27.

36. Nucleotide BLAST: Search nucleotide databases using a nucleotide query. https://blast.ncbi.nlm.nih.gov/Blast.cgi?PROGRAM=blastn\&PAGE TYPE= BlastSearch\&LINK_LOC=blasthome. Accessed 15 Jul 2021.

37. Protein BLAST: search protein databases using a protein query. https:// blast.ncbi.nlm.nih.gov/Blast.cgi?PROGRAM=blastp\&PAGE_TYPE=Blast Search\&LINK LOC=blasthome. Accessed 15 Jul 2021.

38. Pfam: Home page. https://pfam.xfam.org/. Accessed 15 Jul 2021.

39. PHYRE2 Protein Fold Recognition Server. http://www.sbg.bio.ic.ac.uk/ phyre2/html/page.cgi?id=index. Accessed 15 Jul 2021

40. Kelly CR, Kahn S, Kashyap P, Laine L, Rubin D, Atreja A, et al. Update on fecal microbiota transplantation 2015: indications, methodologies, mechanisms, and outlook. Gastroenterology. 2015;149.

41. ARAGORN detects tRNA, mtRNA and tmRNA genes. http://www.ansikte. se/ARAGORN/. Accessed 15 Jul 2021.

42. Laslett D. ARAGORN, a program to detect tRNA genes and tmRNA genes in nucleotide sequences. Nucleic Acids Res. 2004;32.

43. iTOL: Interactive Tree Of Life. https://itol.embl.de/. Accessed 15 Jul 2021.

44. Letunic I, Bork P. Interactive Tree of Life (iTOL) v4: recent updates and new developments. Nucleic Acids Res. 2019:47.

45. Seibt KM, Schmidt T, Heitkam T. FlexiDot: highly customizable, ambiguityaware dotplots for visual sequence analyses. Bioinformatics. 2018;34.

46. Adriaenssens E, Brister JR. How to Name and Classify Your Phage: An Informal Guide. Viruses. 2017;9.

47. ICTV. https://talk.ictvonline.org/files/master-species-lists/m/msl/9601. Accessed 15 Jul 2021

48. Anany H, Switt AIM, De Lappe N, Ackermann H-W, Reynolds DM, Kropinski AM, et al. A proposed new bacteriophage subfamily: "Jerseyvirinae." Arch Virol. 2015:160.

49. Adriaenssens EM, Edwards R, Nash JHE, Mahadevan P, Seto D, Ackermann $\mathrm{H}-\mathrm{W}$, et al. Integration of genomic and proteomic analyses in the classification of the Siphoviridae family. Virology. 2015;477.

50. Turner D, Hezwani M, Nelson S, Salisbury V, Reynolds D. Characterization of the Salmonella bacteriophage vB_SenS-Ent1. J Gen Virol. 2012;93.

51. Kang H-W, Kim J-W, Jung T-S, Woo G-J. wksl3, a New biocontrol agent for Salmonella enterica serovars enteritidis and typhimurium in foods: characterization, application, sequence analysis, and oral acute toxicity study. Appl Environ Microbiol. 2013;79.

52. Lu M, Liu H, Lu H, Liu R, Liu X. Characterization and genome analysis of a novel salmonella phage vB_SenS_SE1. Curr Microbiol. 2020;77.

53. Olsen NS, Hendriksen NB, Hansen LH, Kot W. A new high-throughput screening method for phages: enabling crude isolation and fast identification of diverse phages with therapeutic potential. PHAGE. 2020;1.

54. Vikram A, Woolston J, Sulakvelidze A. Phage biocontrol applications in food production and processing. Curr Issues Mol Biol. 2021.

55. García P, Martínez B, Obeso JM, Rodríguez A. Bacteriophages and their application in food safety. Lett Appl Microbiol. 2008:47. 
56. Galié S, García-Gutiérrez C, Miguélez EM, Villar CJ, Lombó F. Biofilms in the food industry: Health Aspects and Control Methods. Front Microbiol. 2018;9.

57. Gigante A, Atterbury RJ. Veterinary use of bacteriophage therapy in intensively-reared livestock. Virol J. 2019;16.

58. Ross A, Ward S, Hyman P. More is better: selecting for broad host range bacteriophages. Front Microbiol. 2016;7.

59. Davies E V., Winstanley C, Fothergill JL, James CE. The role of temperate bacteriophages in bacterial infection. FEMS Microbiol Lett. 2016;363.

60. Harada LK, Silva EC, Campos WF, Del Fiol FS, Vila M, Dąbrowska K, et al. Biotechnological applications of bacteriophages: State of the art. Microbiol Res. 2018;212-213.

61. EFSA Panel on Additives, or Substances used in Animal Feed (FEEDAP) P, Bampidis V, Azimonti G, Bastos M de L, Christensen H, et al. Safety and efficacy of a feed additive consisting on the bacteriophages PCM F/00069, PCM F/00070, PCM F/00071 and PCM F/00097 infecting Salmonella Gallinarum B/00111 (Bafasal ${ }^{\circledR}$ ) for all avian species (Proteon Pharmaceuticals S.A.). EFSA J. 2021;19:e06534.

62. Santos SB, Kropinski AM, Ceyssens P-J, Ackermann H-W, Villegas A, Lavigne R, et al. Genomic and proteomic characterization of the broad-hostrange salmonella phage PVP-SE1: creation of a new phage genus. J Virol. 2011;85.

63. Santos SB, Fernandes E, Carvalho CM, Sillankorva S, Krylov VN, Pleteneva EA, et al. Selection and characterization of a multivalent Salmonella phage and its production in a nonpathogenic Escherichia coli strain. Appl Environ Microbiol. 2010;76.

64. Tiwari BR, Kim S, Kim J. Complete genomic sequence of salmonella enterica serovar enteritidis phage SE2. J Virol. 2012;86:7712-7712.
65. Hanna LF, Matthews TD, Dinsdale EA, Hasty D, Edwards RA. Characterization of the ELPhiS prophage from salmonella enterica serovar enteritidis strain LK5. Appl Environ Microbiol. 2012;78.

66. Berngruber TW, Weissing FJ, Gandon S. Inhibition of superinfection and the evolution of viral latency. J Virol. 2010;84.

67. Sabzali S, Bouzari M. Isolation, identification and some characteristics of two lytic bacteriophages against Salmonella enterica serovar Paratyphi $B$ and S. enterica serovar Typhimurium from various food sources. FEMS Microbiol Lett. 2021:368.

68. Pickard D, Thomson NR, Baker S, Wain J, Pardo M, Goulding D, et al. Molecular characterization of the Salmonella enterica Serovar Typhi ViTyping Bacteriophage E1. J Bacteriol. 2008;190.

69. Stoddard BL. Homing endonuclease structure and function. Q Rev Biophys. 2006;38.

70. Kwan T, Liu J, DuBow M, Gros P, Pelletier J. The complete genomes and proteomes of 27 Staphylococcus aureus bacteriophages. Proc Natl Acad Sci. 2005;102

71. Santos SB, Carvalho C, Azeredo J, Ferreira EC. Population dynamics of a salmonella lytic phage and its host: implications of the host bacterial growth rate in modelling. PLoS One. 2014;9.

72. National Center for Biotechnology Information. https://www.ncbi.nlm. nih.gov/. Accessed 15 Jul 2021.

\section{Publisher's Note}

Springer Nature remains neutral with regard to jurisdictional claims in published maps and institutional affiliations.
Ready to submit your research? Choose BMC and benefit from:

- fast, convenient online submission

- thorough peer review by experienced researchers in your field

- rapid publication on acceptance

- support for research data, including large and complex data types

- gold Open Access which fosters wider collaboration and increased citations

- maximum visibility for your research: over $100 \mathrm{M}$ website views per year

At $\mathrm{BMC}$, research is always in progress.

Learn more biomedcentral.com/submissions 